\title{
Monte Carlo Computation of Optimal Portfolios in Complete Markets
}

\author{
Jaksa Cvitanić * \\ Levon Goukasian ${ }^{\dagger}$ \\ Fernando Zapatero
}

First draft: January 2000

This draft: January 2000

\begin{abstract}
We introduce a method that relies exclusively on Monte Carlo simulation in order to compute optimal portfolios. Our method is completely general and only require complete markets and knowledge of the dynamics of the security processes. It can be applied regardless or the number of factors and of whether the agent derives utility from intertemporal consumption, terminal wealth or both. We perform some comparative statics.
\end{abstract}

*Department of Mathematics, USC, Los Angeles 90089. Ph: (213) 740-3794. Fax: (213) 7402424. E-mail: cvitanic@math.usc.edu

${ }^{\dagger}$ Department of Mathematics, USC, Los Angeles 90089. Ph: (213) 740-3761. Fax: (213) 7402424. E-mail: goukasia@usc.edu

${ }^{\ddagger}$ FBE, Marshall School of Business, USC, Los Angeles, CA 90089. Ph: (213) 740-6538. Fax: (213) 740-6650. E-mail: fzapatero@sba.usc.edu 


\section{Introduction}

The derivation of the optimal portfolio of a rational investor is a central problem in asset pricing. Although the interest of closed form solutions that would allow to derive equilibrium implications is obvious, the increase in computational power along with the lack of closed form solutions for many interesting cases have triggered an interest in numerical methods as a possible answer to the problem. In this paper we suggest a method purely based on Monte Carlo simulation that allows to solve the problem in complete markets.

Merton (1971) introduced methodology to attack the problem of a rational investor with time additive preferences that chooses how to allocate her wealth between consumption and the existing securities. In his setting, computation of optimal consumption and investment strategies requires the solution of a PDE. However, that PDE only has a closed form solution in a handful of cases.

Karatzas, Lehozcky and Shreve (1987) and Cox and Huang (1989) introduced martingale methods to solve the problem of an utility optimizing investor. Martingale methods allow to consider more general settings than dynamic programming methods and in general allow to compute the optimal consumption policy of the investor. But optimal portfolios, in general, cannot be computed in closed form when martingale methods are used.

A large number of papers have recently undertaking the problem of computation of optimal portfolios. Campbell and Viceira (1999) and Barberis (1998) use numerical approximations to find optimal portfolios in a discrete time setting. In continuous time, Kim and Omberg (1996) solve the PDE in closed form for a specific parameterization of the model. Liu (1998) finds a closed form solution for a general class of parameterizations for an agent whose utility depends only on terminal wealth. Wachter (1999) solves also a specific case but that allows for intertemporal consumption. Brennan, Schwartz and Lagnado (1997) and Xia (1999) solve numerically the PDE also for specific (but more general) parameterizations of the utility function. Finally, Detemple, Garcia, Rindisbacher (1999) compute the Malliavin derivatives of the processes and then use Monte Carlo simulation in order to retrieve the optimal portfolio.

In this paper we introduce a pure Monte Carlo simulation approach very easy to implement and that can be applied whenever two conditions are met (this two conditions are also required by the method introduced in Detemple, Garcia, Rindisbacher (1999):

- Markets are complete, that is, the number of non-redundant stocks and the number of Brownian motion processes that explain the uncertainty of the economy are equal.

- We know the dynamics of all the processes involved (the expanded opportunity set is markovian. 
The method we introduce here can be applied to any type of time additive utility function and any parameterization of the security processes, regardless of whether the agent derives utility from final wealth, intertemporal consumption or both and regardless of the number of Brownian motion processes that explain the uncertainty of the economy. The advantage of Monte Carlo simulation is that it is very easy to implement and converges very fast. Monte Carlo simulation has been increasingly popular to price derivatives since its introduction in finance by Boyle (1977). However, it had not been considered as a tool to solve optimal portfolios until the work of Detemple, Garcia and Rindisbacher (1999).

We propose here a method purely based on Monte Carlo simulation. We use the fact that the optimal portfolio of the investor is part of the standard deviation of the optimal wealth process. Monte Carlo simulation is particularly appropriate to compute expectations and we use this property to compute the second moment (the expected value of the squared change in the wealth level).

The structure of the paper is as follows. In Section 2 we describe the setting and give an intuition of the method. In Section 3 we apply the general idea to the computation of an optimal portfolio. In Section 4 we do some exercises and perform some comparative statics. In Section 5 we explain the extension of the method to the multifactor case. We close the paper with some conclusions.

\section{General Method}

\section{$2.1 \quad$ Securities}

Here we describe the financial assets the investor can choose among. In order to illustrate the method we will define some specific, although fairly general dynamics. As it will be clear later the method is not restricted to the set of prices defined here. In order to simplify the notation, we consider real prices, expressed in terms of the unique consumption good. There are two types of securities. First, there are $n$ stocks whose price satisfies the following dynamics,

$$
\frac{d S_{t}^{i}}{S_{t}^{i}}=\mu_{t}^{i} d t+\left(\sigma_{t}^{i}\right)^{\top} d W_{t}
$$

where $W$ is a vector of $n$ independent standard Brownian motion processes. Realizations of these $n$ Brownian motion processes define the path followed by the economy. Additionaly, $\mu^{i}$ and $\sigma^{i}$ represent the drift and volatility of the stock process $i$ and are possibly stochastic (we discuss their dynamics later). The second type of security is a bond whose price $B$ evolves according to the following dynamics,

$$
d B_{t}=B_{t} r_{t} d t
$$

where $r$ is the possibly stochastic interest rate (we discuss its dynamics later). 
Uncertainty in this economy is given by the realizations of the $n$ dimensional Brownian motion process. We assume that the number of stocks and the numer of Brownian motion processes is the same. Besides, we assume that the matrix $\Sigma$ formed by stacking the $n \sigma^{i}$ vectors is non-singular at every point in time $t$ : this is equivalent to assuming that markets are complete. In order to simplify the notation, we will assume (w.l.g.) throughout the paper that $n=1$ and therefore, $\Sigma=\sigma$. In the last section of the paper we consider the case of $n=2$ (and $n>2$ would be analogous).

We now consider the dynamics of the different parameters of the model. We first define the market price of risk $\theta$ as

$$
\theta=\frac{\mu-r}{\sigma}
$$

As we mentioned above, we consider the possibility of $\mu, r$ and $\sigma$ stochastic. The only restriction that we impose is that all the parameters, plus those of any existing state variable depend on the $n$ dimensional Brownian motion process that describes the uncertainty of this economy. The dependence on the Brownian motion process can be of any type as long as it is known. In summary, we only require the expanded opportunity set to be markovian. In this paper we will restrict to the following dynamics. With respect to the interest rate, we assume that it satisfies the following dynamics:

$$
d r_{t}=\left(a_{r}+b_{r}\left(r_{t}\right)^{l_{r}}+c_{r}\left(\theta_{t}\right)^{p_{r}}\right) d t+\left(d_{r}+f_{r}\left(r_{t}\right)^{q_{r}}+g_{r}\left(\theta_{t}\right)^{v_{r}}\right) d W_{t}
$$

where $a_{r}, b_{r}, c_{r}, d_{r}, f_{r}, g_{r}, l_{r}, p_{r}, q_{r}$ and $v_{r}$ are constant. With respect to $\theta$, we assume that it satisfies

$$
d \theta_{t}=\left(a_{\theta}+c_{\theta}\left(\theta_{t}\right)^{p_{\theta}}\right) d t+\left(d_{\theta}+g_{\theta}\left(\theta_{t}\right)^{v_{\theta}}\right) d W_{t}
$$

where $a_{\theta}, c_{\theta}, d_{\theta}, g_{\theta}, p_{\theta}$ and $v_{\theta}$ are constant. A subset of the previous dynamics are the "affine"models studied in Duffie, Pan and Singleton (2000). In fact, the previous dynamics, although fairly general, are still restrictive. Our approach is completely general in a markovian setting and could deal, for example, with a setting were all the parameters of (4) and (5) where stochastic, as long as they depended on the Brownian motion process and the form of the dynamics were known.

\subsection{General Idea}

We now explain the general idea of the method we will use to compute the optimal portfolio of the individual. In the previous economic setting, consider the expression

$$
C_{t}=E\left[\int_{t}^{T} f\left(r_{s}, \theta_{s}, W_{s}\right) d s \mid \mathcal{F}_{t}\right]
$$

where the information up to moment $t$, represented by $\mathcal{F}_{t}$, is the path of the Brownian motion process up to $t$. Equation (6) satisfies a stochastic differential equation of the type

$$
d C_{t}=\alpha_{t} d t+\nu_{t} d W_{t}
$$


where $\alpha$ and $\nu$ are again possibly stochastic and path-dependent.

Although in general a closed form expression for $\nu$ does not exist, the computation of that parameter is the key in many problems in finance, like hedging of contingent claims or (the problem we consider in this paper) the optimal portfolio of an utility maximizing investor.

In this paper we suggest to use Monte Carlo simulation in order to compute the process $\nu$ (and, therefore, the optimal portfolio of the individual). The method we introduce here can be applied whenever Monte Carlo simulation is possible and two requirements are satisfied: complete markets and markovian expanded opportunity set (regardless of the number of parameters). Monte Carlo simulation has the advantage that it is very easy to implement and converges very quickly.

Monte Carlo simulation was introduced in finance for the pricing of derivatives by Boyle (1977). Boyle, Broadie and Glasserman (1997) offer a detailed survey of the application of Monte Carlo simulation to the pricing of derivatives. In fact, the use of numerical methods in finance has been restricted very recently to the pricing and hedging of derivatives. Only recently numerical methods have started to be used as a way to solve the problem of finding the optimal portfolio. We mention in discrete time, Campbell and Viceira (1999) and Barberis (1998). In continuous time, Brennan, Schwartz and Lagnado (1997) and Xia (1999) use numerical methods to solve the PDE's that result from the dynamic approach. Detemple, Garcia and Rindisbacher (1999) use Monte Carlo simulation combined with the computation of the Malliavan derivatives. In this paper we introduce a method based exclusively on Monte Carlo simulation. The advantage of Monte Carlo simulation over other numerical methods is that it is very easy to implement and converges very quickly.

When Monte Carlo simulation is applied to financial problems, an expression of the type of (1) is discretized in the following way,

$$
S_{t+\Delta t}-S_{t}=S_{t}\left(\mu_{t} \Delta t+\sigma_{t} z_{t}\right)
$$

where $z$ is a quasi-random number drawn from a (computer generated through some type of numerical algorithm) hypothetical normal distribution with zero mean and $\sqrt{t}$ standar deviation. A time horizon $T$ is divided in $N$ intervals of size $\Delta t$ and by generating $N$ values $z$ we will have a discretized version of a possible path of $S$.

Consider the problem of estimating numerically the value $C$ of equation (6): a large numbers of paths of $W$ will be simulated and used in the dynamics of all the relevant processes in the form explained above; in order to compute the expected value of (6) the average of all of the paths will be taken in order to derive $C$. Here we suggest to use the same technique in order to derive $\nu$, the volatility term of the expression (7). From (6), the volatility $\nu$ is $^{1}$

$$
\nu_{t}=\lim _{\Delta t \rightarrow 0}\left(E\left[\frac{\left(C_{t+\Delta t}-C_{t}-\alpha_{t} \Delta t\right)^{2}}{\Delta t} \mid \mathcal{F}_{t}\right]\right)^{1 / 2}
$$

\footnotetext{
${ }^{1}$ This is a heuristic derivation. For a formal treatment of the "quadratic variation" see Karatzas and Shreve (1993)).
} 


$$
=\lim _{\Delta t \rightarrow 0}\left(E\left[\frac{\left(C_{t+\Delta t}-C_{t}\right)^{2}}{\Delta t} \mid \mathcal{F}_{t}\right]\right)^{1 / 2}
$$

We can ignore the effect of the drift $\alpha$ becauseit multiplies $\Delta t$, that converges to 0 and does it faster than in the denominator because the numerator is squared. Alternatively, we can compute $\nu$ as,

$$
\begin{aligned}
\nu_{t} & =\lim _{\Delta t \rightarrow 0} E\left[\frac{\left(C_{t+\Delta t}-C_{t}\right)\left(W_{t+\Delta t}-W_{t}\right)}{\Delta t} \mid \mathcal{F}_{t}\right] \\
& =\lim _{\Delta t \rightarrow 0} E\left[\frac{\left(C_{t+\Delta t}-C_{t}\right)\left(z_{t}\right)}{\Delta t} \mid \mathcal{F}_{t}\right]
\end{aligned}
$$

where $z_{t}$ is the quasi-random number discussed above. Informally, in (9) we compute the standard deviation of the stochastic process while in (10) we compute the covariance between $C$ and the Brownian motion process $W$ (which, clearly, is also $\nu$ ).

As we will see, however, the expression in (10) is more convenient when there is more than one Brownian motion process (the "multifactor" case that we will consider in the last section of the paper).

At moment $t$ we know the value $C_{t}$ but in order to compute numerically the expression in (10) we need to generate a number of values of $C_{t+\Delta t}$ but we do not know the dynamics of $C$ (that is in fact the problem we are trying to solve). However, from (6) we know that $C_{t+\Delta t}$ is the expected value of some function of the parameters of the model (that depend on the path of the Brownian motion process) whose dynamics we know. This is the fact that we will exploit and will allow us to compute optimal portfolios. We explain the exact procedure in next section.

\section{Computation of the Optimal Portfolio}

We consider the problem of a rational, utility maximizing, investor. The utility of this investor is the result of a bequest target, intertemporal consumption or both. There is a single consumption good that we will use as numeraire. Individuals receive an initial endowment in units of the consumption good that they can either consume or invest in the financial markets.

In order to simplify the presentation, we will focus in the two following problems,

$$
\begin{aligned}
U\left(X_{t}\right) & =\max _{\pi} E\left[e^{-\delta(T-t)} \frac{X_{T}^{\gamma}}{\gamma} \mid \mathcal{F}_{t}\right] \\
U\left(X_{t}\right) & =\max _{(\pi, c)} E\left[\int_{t}^{T} e^{-\delta(s-t)} \frac{c_{s}^{\gamma}}{\gamma} d s \mid \mathcal{F}_{t}\right]
\end{aligned}
$$

where $\pi$ represents the trading strategy (to be described below) and $X$ is the wealth level of the investor, $\delta$ is the subjective discount factor (that in order to simplify the notation we will assume constant but is not necessary for our method). The initial, 
exogenous wealth level of the investor is $X_{0}$. In this paper, for simplicity, we will only consider CRRA utilities with $\gamma$ as the parameter that characterizes the degree of risk aversion. Again, as pointed above, our method is completely general and can be applied to any type of utility function. However, we need utilities to be time additive. Within this class of utility functions, our method allows us to solve any case.

The investor can allocate her wealth either in consumption $c$ or in any of the securities described above. We denote by $\pi$ the amount of wealth invested in the stock. The wealth process $X$ of the investor satisfies,

$$
d X_{t}=\left(\pi_{t} \mu_{t}+\left(\pi-X_{t}\right) r_{t}-c_{t}\right) d t+\pi_{t} \sigma_{t} d W_{t}
$$

The previous problem was first considered in continuous time by Merton (1979), using dynamic programming. More recently, Karatzas, Lehoczky and Shreve (1987) and Cox and Huang (1991), introduced martingale methods that allow to solve the problem using lagrange multipliers (as an static problem). Following the approach of these two papers and using the notation introduced in (3), we can write (13) as

$$
\begin{aligned}
d X_{t} & =\left(r_{t} X_{t}-c_{t}\right) d t+\pi_{t}\left(\sigma_{t} d W_{t}+\theta_{t} d t\right) \\
& =\left(r_{t} X_{t}-c_{t}\right) d t+\pi_{t} \sigma_{t} d \tilde{W}_{t}
\end{aligned}
$$

where $\tilde{W}$ is a Brownian motion process with respect to $Q$, the "equivalent risk-neutral probability."We define, the process,

$$
\xi_{t}=\exp \left(-\frac{1}{2} \int_{0}^{t} \theta_{s}^{2} d s-\int_{0}^{t} \theta_{s} d W_{s}\right)
$$

The present value of this process represents the continuous time Arrow-Debreu prices. The problem of the investor is equivalent to the maximization of equation (11) subject to:

$$
E^{Q} e^{-\int_{t}^{T} r_{s} d s} X_{T}=E \xi_{T} e^{-\int_{t}^{T} r_{s} d s} X_{T}=X_{0}
$$

or, respectively, maximization of equation (12) subject to

$$
E^{Q} \int_{t}^{T} e^{-\int_{t}^{s} r_{u} d u} c_{s} d s=E \xi_{T} \int_{t}^{T} e^{-\int_{t}^{s} r_{u} d u} c_{s} d s=X_{0}
$$

where $\xi$ is given by equation (15). The dynamic problem has now become an static problem. Using standard optimization techniques we find that the respective optimal final wealth and optimal consumption strategies are given by,

$$
\begin{aligned}
X_{T}^{*} & =\left(y e^{\int_{0}^{T}\left(\delta-r_{s}\right) d s} \xi_{T}\right)^{\frac{1}{\gamma-1}} \\
c_{t}^{*} & =\left(y \int^{\int_{0}^{T}\left(\delta-r_{s}\right) d s} \xi_{t}\right)^{\frac{1}{\gamma-1}}
\end{aligned}
$$

where $y$ is the lagrange multiplier, the scaling constant that guarantees that the budget constraints (16) and (17) are satisfied. 
It is by now standard that in the complete markets specification that we consider here the wealth process $X$ can also be expressed as an expectation of the type described in equation (6). More explicitely, the value of the wealth process at every point in time is the expected discounted value of future consumption and/or terminal wealth under the equivalent "risk-neutral" probability measure that depends on $\theta$ (this is in fact the result used to derive the budget constraints of (16) and (17):

$$
X_{t}=E^{Q}\left[e^{-\int_{t}^{T} r_{s} d s} X_{T} \mid \mathcal{F}_{t}\right]=\frac{1}{\xi_{t}} E\left[\xi_{T} e^{-\int_{t}^{T} r_{s} d s} X_{T} \mid \mathcal{F}_{t}\right]
$$

in the case of utility from final wealth and

$$
X_{t}=E^{Q}\left[\int_{t}^{T} e^{-\int_{t}^{s} r_{u} d u} c_{s} d s \mid \mathcal{F}_{t}\right]=\frac{1}{\xi_{t}} E\left[\xi_{T} \int_{t}^{T} e^{-\int_{t}^{s} r_{u} d u} c_{s} d s \mid \mathcal{F}_{t}\right]
$$

in the case of utility from intertemporal consumption, with $\xi$ given by equation (15). But the right hand sides of (20) and (21)are expressions of the type of (6) and, therefore, satisfy

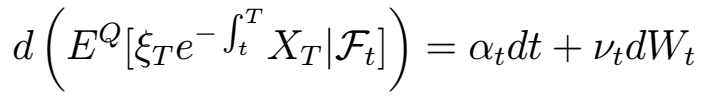

for (20) and similarly for (21) but comparing (13) and (22) we conclude that the optimal portfolio is given by

$$
\pi_{t}^{*}=\left(\sigma_{t}\right)^{-1} \nu_{t}
$$

and, therefore, by retrieving numerically $\nu$ using the method explained in the previous section, we can derive the optimal portfolio strategy.

More explicitely, from (10),

$$
\begin{aligned}
\nu_{t} & =\lim _{\Delta t \rightarrow 0} E\left[\frac{\left(X_{t+\Delta t}-X_{t}\right)\left(W_{t+\Delta t}-W_{t}\right)}{\Delta t} \mid \mathcal{F}_{t}\right] \\
& =\lim _{\Delta t \rightarrow 0} E\left[\frac{\left(X_{t+\Delta t}-X_{t}\right)\left(z_{t}\right)}{\Delta t} \mid \mathcal{F}_{t}\right]
\end{aligned}
$$

where $z_{t}$ is the computer generated quasi-random number that we discussed in the previous section (for more on that see Press, Teukolsky, Vetterling and Flannery (1992)).

Therefore, the specific procedure is as follows. At moment $t$ we know $X_{t}$. In order to compute (24) we need to compute, for each realization of the (computer generated) Brownian motion process $z$ the corresponding wealth value $X_{t+\Delta t}$. But,

$$
\begin{aligned}
X_{t+\Delta t} & =E^{Q}\left[e^{-\int_{t+\Delta t}^{T} r_{s} d s} X_{T} \mid \mathcal{F}_{t+\Delta t}\right] \\
& =E\left[\frac{\xi_{T}}{\xi_{t+\Delta t}} e^{-\int_{t+\Delta t}^{T} r_{s} d s} X_{T} \mid \mathcal{F}_{t+\Delta t}\right] \\
& =E\left[\frac{\xi_{T}}{\xi_{t+\Delta t}} e^{-\int_{t+\Delta t}^{T} r_{s} d s}\left(y e^{\int_{0}^{T}\left(\delta-r_{s}\right) d s} \xi_{T}\right)^{\frac{1}{\gamma-1}} \mid \mathcal{F}_{t+\Delta t}\right]
\end{aligned}
$$


for the the case of utility from final wealth, where we have used (18) and

$$
\begin{aligned}
X_{t+\Delta t} & =E^{Q}\left[\int_{t+\Delta t}^{T} e^{-\int_{t+\Delta t}^{s} r_{u} d u} c_{s} d s \mid \mathcal{F}_{t+\Delta t}\right] \\
& =E\left[\frac{\xi_{T}}{\xi_{t+\Delta t}} \int_{t+\Delta t}^{T} e^{-\int_{t+\Delta t}^{s} r_{u} d u} c_{s} d s \mid \mathcal{F}_{t+\Delta t}\right] \\
& =E\left[\frac{\xi_{T}}{\xi_{t+\Delta t}} \int_{t+\Delta t}^{T} e^{-\int_{t+\Delta t}^{s} r_{u} d u}\left(y e^{\int_{0}^{s}\left(\delta-r_{u}\right) d u} \xi_{s}\right)^{\frac{1}{\gamma-1}} d s \mid \mathcal{F}_{t+\Delta t}\right]
\end{aligned}
$$

in the case of utility from intertemporal consumption, where we have used (19). The right hand side of (25) and (26) will not have in general a closed form solution (exceptions are the logarithmic utility case $\gamma=1$ and the affine models considered in Duffie, Pan and Singleton (2000)). However we can use Monte Carlo simulation to compute that right-hand side. In order to do that we need to know the dynamics of the processes involved. With respect to $\xi$, from (15) it satisfies,

$$
\frac{d \xi_{t}}{\xi_{t}}=-\theta_{t} d W_{t}
$$

and the dynamics of $r$ and $\theta$ are given by (4) and (5). At moment $t$ we know $X_{t}$, $r_{t}$, and $\theta_{t}$. We discretize these processes as explained in (8) and generate a large number of paths for each of them, compute the expressions in the right hand side of (25) and (26) and average over them in order to get our estimate of $X_{t+\Delta t}$ for a given realization of the (computer generated) Brownian motion $z$. Then we generate another Brownian motion process and get a second estimate of $X_{t+\Delta t}$. After a number of estimates of $X_{t+\Delta t}$ have been generated, we average over all of them in order to compute (24).

In summary, we use a two-step procedure:

1. We are at moment $t$ and know $X_{t}, r_{t}, \theta_{t}$ and $\xi_{t}$. We observe (generate with the computer) a realization of the Brownian motion process, say $z_{1}$. Using the dynamics of $r, \theta$ and $\xi$ we upgrade their values to $r_{t+\Delta t}\left(z_{1}\right), \theta_{t+\Delta t}\left(z_{1}\right)$ and $\xi_{t+\Delta t}\left(z_{1}\right)$ and compute $X_{t+\Delta t}\left(z_{1}\right)$ using (25) or (26). With the notation we mean that we are at moment $t$ and we will compute a value of the wealth process at $t+\Delta t$ contingent on getting a realization of the Brownian motion process equal to $z_{1}$ at $t$.

2. We generate a second value for the Brownian motion process at $t$, say $z_{2}$ and compute $X_{t+\Delta t}\left(z_{2}\right)$. We repeat the procedure $N$ times and collect the values $X_{t+\Delta t}\left(z_{i}\right), i=1, \ldots N$. Then we use (24) and compute,

$$
\frac{1}{N} \sum_{i=1}^{N} \frac{\left(X_{t+\Delta t}\left(z_{i}\right)-X_{t}\right) z_{i}}{\Delta t}
$$


Clearly, this procedure is independent of the type of utility function, whether the investor derives utility only from terminal wealth, from intertemporal consumption or from both and of the dynamics of the stochastic processes involved (as long as those dynamics are known).

In the next section we analyze some results for different values of the parameters of the model.

\section{Analysis of results}

The basic model we study is a simplification of the general model presented in section 2. With respect to the interest rate, we consider the Cox, Ingersoll and Ross (1985) dynamics, that is,

$$
d r_{t}=\kappa_{r}\left(\bar{r}-r_{t}\right) d t-\sigma_{r} \sqrt{r_{t}} d W_{t}
$$

For the equity premium $\theta$ we first consider a simple mean-reverting process with constant volatity,

$$
d \theta_{t}=\kappa_{\theta}\left(\bar{\theta}-\theta_{t}\right) d t+\sigma_{\theta} d W_{t}
$$

This is in fact the model considered in Detemple, Garcia and Risdinbacher (1999). They calibrate this model and find the following values for the parameters,

$$
\begin{gathered}
\bar{r}=.06, \quad \sigma_{r}=.0364, \quad \kappa_{r}=.0824 \kappa_{\theta}=.6950, \quad \bar{\theta}=.0871, \\
\sigma_{\theta}=.21, \quad \sigma(t) \equiv .2, \quad r(0)=.06, \quad \theta(0)=.1 .
\end{gathered}
$$

With respect to the utility function, Detemple, Garcia and Risdinbacher (1999) only consider the case of utility from terminal wealth. We will consider both cases as expressed in equations (11) and (12). In the case of utility from terminal wealth, with time horizon $T=1$, risk-aversion characterized by $\gamma=-1$ and initial wealth $X_{0}=1$ (so that the portfolio can be interpreted as the proportion of current wealth invested in the risky stock) which is the case considered in Detemple, Garcia and Risdinbacher (1999) we obtain an optimal investment in the risky security of $\pi^{*}=0.252$.

In Table 1 we consider the sensitivity of the portfolio both to changes in riskaversion and horizon. Risk-aversion is measured by the parameter $\gamma$ : the lower $\gamma$ (which has to be smaller than 1 to guarantee the concavity of the utility function) the more risk averse the individual. The case $\gamma=0$ corresponds to an agent with logarithmic utility. In that case the problem has closed-form solution and the optimal portfolio is $\frac{\theta}{\sigma}$. We consider both the case in which the individual only draws utility from terminal wealth (TW) and when the individual draws utility from intertemporal consumption (IC). As expected, when both the horizon and risk aversion increase, the investor allocates a larger proportion of wealth to the risky asset. The investor allocates a smaller proportion of wealth to the risky security when she derives utility from intertemporal consumption. 
In Tables 2 and 3 we consider changes in $\kappa_{r}$, the speed of mean-reversion, $\sigma_{r}$, the constant component of the volatility of the interest rate and $\sigma_{\theta}$, the constant component of the volatility of the equity premium. Increase in mean-reversion of the interest rate decreases holdings of the risky security since there is less need to hedge given the larger stability of the interes rate. When the volatility of the interest rate increases the agent invests more in the risky security (which is negatively correlated to the interest rate). When volatility of the risk premium process increases, the agent invests less in the risky security (since it becomes less attractive).

Finally, we consider the following alternative dynamics of the interest rate and the equity premium process,

$$
\begin{aligned}
d r_{t} & =\kappa_{r}\left(\bar{r}-r_{t}\right) d t-\sigma_{r}\left(r_{t}\right)^{v_{r}} d W_{t} \\
d \theta_{t} & =\kappa_{\theta}\left(\bar{\theta}-\theta_{t}\right) d t+\sigma_{\theta}(\theta)^{v_{\theta}} d W_{t}
\end{aligned}
$$

For $v_{r} \neq 0.5$ and/or $v_{\theta} \neq 0$ the problem does not belong to the class of affine models anymore. In Tables 4 and 5 we study effect of changes in these parameters. When $v_{r}$ changes the volatility of the interest rate process is not proportional to the volatility of the interest rate and the time effect is lessened. When $v_{\theta}$ increases, however, the proportion of wealth invested in the risky security increases. In fact, the investment allocation seems to be very sensitive to changes in this parameter.

Overall, however, it seems that, for time additive CRRA preferences (which are the standard in the literature) portfolio allocation does not change very much, unless a very high degree of risk aversion is considered.

\section{$5 \quad$ Multiple Factors}

For ease of notation, in the previous section we have considered the case of a single Brownian motion process. However, as we stated before, the method that we suggest in this paper can be applied regardless of the number of Brownian motion processes that explain the dynamics of the model, as long as we stay in a complete markets setting, that is, the number of stocks is equal to the number of Brownian motion processes and the variance covariance matrix of all the stocks is non-singular.

Suppose, for example that we have two stocks and two independent standard Brownian motion processes $W^{1}$ and $W^{2}$. The price of each of the stock processes $S^{i}, i=1,2$ satisfies

$$
\frac{d S_{t}^{i}}{S_{t}^{i}}=\mu_{t}^{i}+\sigma_{t}^{i 1} d W_{t}^{1}+\sigma_{t}^{i 2} d W^{2}, i=1,2
$$

and we assume that the matrix

$$
\Sigma=\left(\begin{array}{cc}
\sigma_{t}^{11} & \sigma_{t}^{12} \\
\sigma_{t}^{21} & \sigma_{t}^{22}
\end{array}\right)
$$


is non-singular for all $t$.

In this setting, the agent can invest in both securities and, as a result of it, his/her wealth dynamics will be of the type,

$$
d X_{t}=\alpha_{t} d t+\nu_{t}^{1} d W_{t}^{1}+\nu_{t}^{2} d W_{t}^{2}
$$

Of course the optimal portfolio of the individual will be now bi-dimensional and, as in (23) equal to,

$$
\pi^{*}=(\Sigma)^{-1} \nu
$$

where $\nu^{\top}=\left(\nu^{1}, \nu^{2}\right)$.

The implementation of Monte Carlo simulation is analogous to the one-dimensional case, but now we will have to generate two simultaneous series of random numbers $z^{1}$ and $z^{2}$. The discrete version of (33) is

$$
S_{t+\Delta t}^{i}-S_{t}^{i}=S_{t}^{i}\left(\mu_{t}^{i} \Delta t+\sigma_{t}^{i 1} z_{t}^{1}++\sigma_{t}^{i 2} z_{t}^{2}\right), i=1,2
$$

Analogously, we can replicate the paths of the stochastic processes involved, $r, \theta$ and $\xi$. In order to retrieve $\nu^{1}$ and $\nu^{2}$ we use, (this is similar to (24)),

$$
\nu_{t}^{i}=\lim _{\Delta t \rightarrow 0} E\left[\frac{\left(X_{t+\Delta t}-X_{t}\right)\left(z_{t}^{i}\right)}{\Delta t} \mid \mathcal{F}_{t}\right], i=1,2
$$

In order to implement this expression we use a two-step procedure as the one described in the one-dimensional case, but with the following modification: we start at $t$ where we know $X_{t}, r_{t}$ and $\theta_{t}$. We generate one realization of each of the Brownian motion processes that we call $z_{1}^{1}$ and $z_{1}^{2}$ and compute $r_{t+\Delta t}\left(z_{1}^{1}, z_{1}^{2}\right), \theta_{t+\Delta t}\left(z_{1}^{1}, z_{1}^{2}\right)$ and $\xi_{t+\Delta t}\left(z_{1}^{1}, z_{1}^{2}\right)$. Then we compute $X_{t+\Delta t}\left(z_{1}^{1}, z_{1}^{2}\right)$ by replicating the paths of all the stochastic processes involved. Then, we generate another pair of realization of the Brownian motion processes, $z_{2}^{1}$ and $z_{2}^{2}$ and we repeat the process to get $X_{t+\Delta t}\left(z_{2}^{1}, z_{2}^{2}\right)$. We repeat the process $N$ times and approximate each $\nu^{i}$ (whose estimate we denote by $\hat{\nu}^{i}$ ) by computing,

$$
\hat{\nu}^{i}=\frac{1}{N} \sum_{i=1}^{N} \frac{\left(X_{t+\Delta t}\left(z_{1}^{1}, z_{1}^{2}\right)-X_{t}\right) z_{1}^{i}}{\Delta t}, i=1,2
$$

Obviously, the approach would be analogous if we had more than two factors.

\section{Conclusions}

In this paper we introduce a method that relies exclusively on Monte Carlo simulation in order to compute optimal portfolios. Our method is completely general and only require complete markets and knowledge of the dynamics of the security processes. It can be applied regardless or the number of factors and of whether the 
agent derives utility from intertemporal consumption, terminal wealth or both. The implementation is very easy ansd allows us to perform some comparative statics. The method relies on the fact that the optimal portfolio is part of the standard deviation of the wealth process and such standard deviation can be directly estimated. In fact, computing the standard deviation through Monte Carlo simulation has other applications in finance, like the computation of the optimal hedge of an option (see Cvitanić, Goukassian and Zapatero (1999)). 


\section{References}

[1] Barberis, N., 1998, "Investing for the Long Run when Returns are Predictable," Journal of Finance, forthcoming.

[2] Brennan, M., Schwartz, E. and R. Lagnado, 1997, "Strategic Asset Allocation," Journal of Economic Dynamics and Control, 21, 1377- 1403.

[3] Boyle, P., 1977, "Options: a Monte Carlo Approach," Journal of Financial Economics 4, 323-338.

[4] Boyle, P., M. Broadie and P. Glasserman, 1997, "Monte Carlo Methods for Security Pricing," Journal of Economic Dynamics and Control, 21, 1267- 1321.

[5] Campbell, J. and L. Viceira, 1999 "Consumption and Portfolio Decisions When Expected Returns Are Time Varying," Quarterly Journal of Economics 114, 433-495.

[6] Cox, J., and C.F. Huang, 1989, "Optimal Consumption and Portfolio Policies When Asset Prices Follow a Diffusion Process," Journal of Economic Theory 49, $33-83$.

[7] Cox, J., J. Ingersoll and S. Ross, 1985, "A Theory of the Term Structure of Interest Rates," Econometrica, 53, 385-408.

[8] Cvitanić, J., Goukassian, L. and F. Zapatero, 1999, "Hedging with Monte Carlo simulation," working paper, USC.

[9] Detemple, J., Garcia, R. and M. Rindisbacher, 1999, "A Monte Carlo Approach for Optimal Portfolios," working paper, Boston University.

[10] Duffie, D., 1996, Dynamic Asset Pricing Theory, Princeton University Press, 2nd ed, Princeton.

[11] Duffie, D., Pan, J. and K. Singleton, 1999, "Transform Analysis and Asset Pricing for Affine Jump-Diffusions," Econometrica, forthcoming.

[12] Karatzas, I. and S. Shreve, 1992, Brownian Motion and Stochastic Calculus, 2nd ed., Springer-Verlag, New York.

[13] Karatzas, I., J.P. Lehoczky and S.E. Shreve, 1987, "Optimal Portfolio and Consumption Decisions for a 'Small Investor' on a Finite Horizon," SIAM Journal of Control and Optimization 25, 1557-1586.

[14] Kim, T. and E. Omberg, 1996 "Dynamic Nonmyopic Portfolio Behavior," Review of Financial Studies 9, 141-161. 
[15] Liu, J, 1998, "Porfolio Selection in Stochastic Environments," Ph D Thesis, Stanford University.

[16] Merton, R., 1971, "Optimal Consumption and Portfolio Rules in a Continuous Time Model," Journal of Economic Theory 3, 373-413.

[17] Press, W., S. Teukolsky, W. Vetterling and B. Flannery, 1992, Numerical Recipes in $C$ : the Art of Scientific Computing, Cambridge University Press, New York.

[18] Wachter, J., 1999, "Risk Aversion and Allocation to Long-Term bonds," working paper, Harvard University.

[19] Xia, Y., 1999, "Learning about Predictability: The Effect of Parameter Unceratinty on Dynamic Asset Allocation," working paper, UCLA. 
Table 1: Effect of Horizon and Risk Aversion

$$
\begin{gathered}
\bar{r}=0.06 ; \bar{\theta}=0.0871 ; r_{0}=0.06 ; \theta_{0}=0.1 ; \sigma_{0}=0.2 \\
\kappa_{r}=0.824 ; \kappa_{\theta}=0.6950 ; \sigma_{r}=0.0364 ; \sigma_{\theta}=0.21
\end{gathered}
$$

\begin{tabular}{|c|c|c|c|c|c|c|}
\hline & \multicolumn{2}{|c|}{$T=1$} & \multicolumn{2}{c|}{$T=5$} & \multicolumn{2}{c|}{$T=10$} \\
\hline$\gamma$ & IC & TW & IC & TW & IC & TW \\
\hline 0.5 & 1.042 & 1.095 & 1.221 & 1.236 & 1.230 & 1.146 \\
0 & 0.5 & 0.5 & 0.5 & 0.5 & 0.5 & 0.5 \\
-1 & 0.244 & 0.252 & 0.270 & 0.295 & 0.297 & 0.328 \\
-2 & 0.174 & 0.175 & 0.210 & 0.230 & 0.239 & 0.270 \\
-5 & 0.074 & 0.080 & 0.135 & 0.170 & 0.185 & 0.213 \\
-10 & 0.056 & 0.059 & 0.125 & 0.139 & 0.193 & 0.193 \\
\hline
\end{tabular}

Table 2: Effect of Parameter Dynamics for $\gamma=-1$

$$
\begin{gathered}
\bar{r}=0.06 ; \bar{\theta}=0.0871 ; r_{0}=0.06 \\
\theta_{0}=0.1 ; \sigma_{0}=0.2 ; \kappa_{\theta}=0.6950
\end{gathered}
$$

\begin{tabular}{|c|c|c|c|c|c|c|c|c|}
\hline \multicolumn{2}{|c|}{} & \multicolumn{2}{c|}{$T=1$} & \multicolumn{2}{c|}{$T=5$} & \multicolumn{2}{c|}{$T=10$} \\
\hline$\kappa_{r}$ & $\sigma_{r}$ & $\sigma_{\theta}$ & IC & TW & IC & TW & IC & TW \\
\hline 0.0824 & 0.0364 & 0.21 & 0.244 & 0.252 & 0.270 & 0.295 & 0.297 & 0.328 \\
0.12 & 0.0364 & 0.21 & 0.240 & 0.252 & 0.269 & 0.285 & 0.276 & 0.289 \\
0.0824 & 0.05 & 0.21 & 0.257 & 0.257 & 0.282 & 0.311 & 0.274 & 0.297 \\
0.0824 & 0.0364 & 0.3 & 0.234 & 0.243 & 0.262 & 0.283 & 0.280 & 0.298 \\
\hline
\end{tabular}


Table 3: Effect of Parameter Dynamics for $\gamma=-2$

$$
\begin{gathered}
\bar{r}=0.06 ; \bar{\theta}=0.0871 ; r_{0}=0.06 \\
\theta_{0}=0.1 ; \sigma_{0}=0.2 ; \kappa_{\theta}=0.6950
\end{gathered}
$$

\begin{tabular}{|c|c|c|c|c|c|c|c|c|}
\hline \multicolumn{4}{|c|}{} & \multicolumn{2}{c|}{$T=1$} & \multicolumn{2}{c|}{$T=5$} & \multicolumn{2}{c|}{$T=10$} \\
\hline$\kappa_{r}$ & $\sigma_{r}$ & $\sigma_{\theta}$ & IC & TW & IC & TW & IC & TW \\
\hline 0.0824 & 0.0364 & 0.21 & 0.174 & 0.175 & 0.210 & 0.232 & 0.239 & 0.270 \\
0.12 & 0.0364 & 0.21 & 0.175 & 0.176 & 0.194 & 0.205 & 0.234 & 0.248 \\
0.0824 & 0.05 & 0.21 & 0.167 & 0.180 & 0.213 & 0.241 & 0.259 & 0.290 \\
0.0824 & 0.0364 & 0.3 & 0.166 & 0.170 & 0.192 & 0.207 & 0.219 & 0.242 \\
\hline
\end{tabular}

Table 4: Non-affine models with $\gamma=-1$

\begin{tabular}{|c|c|c|c|c|c|c|c|}
\hline \multicolumn{10}{|c|}{$\bar{r}=0.06 ; \bar{\theta}=0.0871 ; r_{0}=0.06 ; \theta_{0}=0.1 ; \sigma_{0}=0.2$} \\
$\kappa_{r}=0.824 ; \kappa_{\theta}=0.6950 ; \sigma_{r}=0.0364 ; \sigma_{\theta}=0.21$ \\
\hline$v_{r}$ & $v_{\theta}$ & IC & TW & IC & TW & IC & TW \\
\hline 0.5 & 0 & 0.244 & 0.252 & 0.270 & 0.295 & 0.297 & 0.328 \\
0.25 & 0 & 0.249 & 0.253 & 0.228 & 0.231 & 0.244 & 0.245 \\
2 & 0 & 0.244 & 0.233 & 0.246 & 0.243 & 0.226 & 0.233 \\
0.5 & 0.5 & 0.246 & 0.260 & 0.286 & 0.308 & 0.322 & 0.346 \\
0.5 & 2 & 0.265 & 0.266 & 0.300 & 0.323 & 0.335 & 0.362 \\
\hline
\end{tabular}




\begin{tabular}{|c|c|c|c|c|c|}
\hline \multicolumn{6}{|c|}{$\begin{array}{c}\text { Table 5: Non-affine models } \\
\text { with } \gamma=-2\end{array}$} \\
\hline \multicolumn{6}{|c|}{$\begin{array}{c}\bar{r}=0.06 ; \bar{\theta}=0.0871 ; r_{0}=0.06 \\
\theta_{0}=0.1 ; \sigma_{0}=0.2 ; \kappa_{r}=0.824 \\
\kappa_{\theta}=0.6950 ; \sigma_{r}=0.0364 ; \sigma_{\theta}=0.21\end{array}$} \\
\hline & & \multicolumn{2}{|c|}{$T=1$} & \multicolumn{2}{|c|}{$T=5$} \\
\hline$v_{r}$ & $v_{\theta}$ & $\mathrm{IC}$ & $\mathrm{TW}$ & $\mathrm{IC}$ & TW \\
\hline 0.5 & 0 & 0.174 & 0.175 & 0.210 & 0.230 \\
\hline 0.25 & 0 & 0.169 & 0.170 & 0.165 & 0.168 \\
\hline 2 & 0 & 0.172 & 0.166 & 0.154 & 0.156 \\
\hline 0.5 & 0.5 & 0.184 & 0.189 & 0.221 & 0.244 \\
\hline 0.5 & 2 & 0.186 & 0.189 & 0.232 & 0.258 \\
\hline
\end{tabular}

